\title{
Article \\ Cerebral Biomarkers and Blood-Brain Barrier Integrity in Preeclampsia
}

\author{
Therese Friis 1,*DD, Anna-Karin Wikström ${ }^{1}$, Jesenia Acurio 2,3, José León 2,3,4 , Henrik Zetterberg 5,6,7,8,9, \\ Kaj Blennow ${ }^{5,6}$, Maria Nelander ${ }^{1}$, Helena Åkerud ${ }^{10}$, Helena Kaihola ${ }^{10}$, Catherine Cluver ${ }^{11}$, Felipe Troncoso ${ }^{2}$, \\ Pablo Torres-Vergara ${ }^{3,12} \mathbb{D}$, Carlos Escudero ${ }^{2,3,+}{ }^{(\mathbb{D}}$ and Lina Bergman $1,11,13,+(\mathbb{D}$
}

1 Department of Women's and Children's Health, Uppsala University, 75185 Uppsala, Sweden; anna-karin.wikstrom@kbh.uu.se (A.-K.W.); maria.nelander@kbh.uu.se (M.N.); lina.bergman@kbh.uu.se (L.B.)

2 Vascular Physiology Laboratory, Department of Basic Sciences, Faculty of Sciences, University of Bío-Bío, Chillán 3810178, Chile; jeseniacurio2@gmail.com (J.A.); joseman1115@gmail.com (J.L.); fetronc@gmail.com (F.T.); cescudero@ubiobio.cl (C.E.)

3 Group of Research and Innovation in Vascular Health (GRIVAS Health), Chillán 3810178, Chile; pabltorr@udec.cl

4 Escuela de Enfermería, Facultad de Salud, Universidad Santo Tomás, Los Ángeles 4441171, Chile

5 Department of Psychiatry and Neurochemistry, Institute of Neuroscience and Physiology, The Sahlgrenska Academy at the University of Gothenburg, 43180 Mölndal, Sweden; henrik.zetterberg@clinchem.gu.se (H.Z.); kaj.blennow@neuro.gu.se (K.B.)

6 Clinical Neurochemistry Laboratory, Sahlgrenska University Hospital, 43180 Mölndal, Sweden

7 Department of Neurodegenerative Disease, UCL Queen Square Institute of Neurology, Queen Square, London WC1E 6BT, UK

8 UK Dementia Research Institute at UCL, London WC1E 6BT, UK

check for updates

Citation: Friis, T.; Wikström, A.-K.; Acurio, J.; León, J.; Zetterberg, H.; Blennow, K.; Nelander, M.; Åkerud, H.; Kaihola, H.; Cluver, C.; et al. Cerebral Biomarkers and Blood-Brain Barrier Integrity in Preeclampsia. Cells 2022, 11, 789. https://doi.org/ 10.3390/cells11050789

Academic Editors: Junie P. Warrington, Ana T. Palei, Mark W. Cunningham and Lorena M. Amaral

Received: 27 December 2021

Accepted: 20 February 2022

Published: 24 February 2022

Publisher's Note: MDPI stays neutral with regard to jurisdictional claims in published maps and institutional affiliations.

Copyright: () 2022 by the authors. Licensee MDPI, Basel, Switzerland. This article is an open access article distributed under the terms and conditions of the Creative Commons Attribution (CC BY) license (https:// creativecommons.org/licenses/by/ $4.0 /)$.
9 Hong Kong Center for Neurodegenerative Diseases, Clear Water Bay, Hong Kong, China

10 Department of Immunology, Genetics and Pathology, Uppsala University, 75185 Uppsala, Sweden; helena.akerud@gynhalsan.se (H.Å.); helena.kaihola@outlook.com (H.K.)

11 Department of Obstetrics and Gynecology, Stellenbosch University, Cape Town 7500, South Africa; cathycluver@hotmail.com

12 Department of Pharmacy, Faculty of Pharmacy, University of Concepción, Concepción 4070386, Chile

13 Department of Obstetrics and Gynecology, Gothenburg University, 41650 Gothenburg, Sweden

* Correspondence: therese.friis@kbh.uu.se; Tel.: +46-18-611-6613

+ These authors contributed equally to this work.

Abstract: Cerebral complications in preeclampsia contribute substantially to maternal mortality and morbidity. There is a lack of reliable and accessible predictors for preeclampsia-related cerebral complications. In this study, plasma from women with preeclampsia $(n=28)$, women with normal pregnancies $(n=28)$ and non-pregnant women $(n=16)$ was analyzed for concentrations of the cerebral biomarkers neurofilament light (NfL), tau, neuron-specific enolase (NSE) and S100B. Then, an in vitro blood-brain barrier (BBB) model, based on the human cerebral microvascular endothelial cell line (hCMEC/D3), was employed to assess the effect of plasma from the three study groups. Transendothelial electrical resistance (TEER) was used as an estimation of BBB integrity. NfL and tau are proteins expressed in axons, NSE in neurons and S100B in glial cells and are used as biomarkers for neurological injury in other diseases such as dementia, traumatic brain injury and hypoxic brain injury. Plasma concentrations of NfL, tau, NSE and S100B were all higher in women with preeclampsia compared with women with normal pregnancies (8.85 vs. $5.25 \mathrm{ng} / \mathrm{L}, p<0.001 ; 2.90 \mathrm{vs}$. $2.40 \mathrm{ng} / \mathrm{L}, p<0.05 ; 3.50$ vs. $2.37 \mu \mathrm{g} / \mathrm{L}, p<0.001$ and 0.08 vs. $0.05 \mu \mathrm{g} / \mathrm{L}, p<0.01$, respectively). Plasma concentrations of NfL were also higher in women with preeclampsia compared with non-pregnant women $(p<0.001)$. Higher plasma concentrations of the cerebral biomarker NfL were associated with decreased TEER $(p=0.002)$ in an in vitro model of the BBB, a finding which indicates that NfL could be a promising biomarker for BBB alterations in preeclampsia.

Keywords: blood-brain barrier; preeclampsia; pregnancy; in vitro studies; cerebral biomarkers; NfL; tau; NSE; S100B 


\section{Introduction}

Preeclampsia affects 3-5\% of all pregnancies and is one of the most common causes of maternal and perinatal morbidity and mortality [1]. Preeclampsia is defined as de novo hypertension after 20 weeks of gestation accompanied by signs of maternal organ dysfunction, such as renal insufficiency, liver dysfunction, neurological features, hematological complications or fetal growth restriction as a sign of uteroplacental dysfunction [2,3]. Annually, more than 70,000 maternal deaths are associated with hypertensive disorders of pregnancy, where cerebral complications due to preeclampsia, such as eclampsia, cerebral edema and cerebral hemorrhage are leading causes of maternal death [3-5]. In the majority of cases, eclampsia is preceded by hypertension and in some cases by neurological symptoms; however, eclampsia can occur before the onset of hypertension and often in the absence of premonitory symptoms [6]. There is a lack of objective biomarkers for cerebral complications to preeclampsia and the underlying pathophysiology remains partly unknown.

The cerebral biomarkers neurofilament light (NfL), tau, neuron-specific enolase (NSE) and S100B are all present within cells of the central nervous system, the last of these in the astrocytic endfeet contributing to the neurovascular unit of the blood-brain barrier (BBB) [7]. They have all been extensively studied in the context of hypoxic or traumatic brain injury, different types of dementia and epilepsy. NfL and tau are both axonal proteins used as biomarkers for neurodegenerative disease [8,9]. In patients with traumatic brain injury, increased concentrations of NfL have been detected in both cerebrospinal fluid (CSF) and peripheral blood, compared with controls [10]. NSE is a glycolytic enzyme mainly found in neurons [11], which has proved useful in prognostication of patients with cardiac arrest and hypoxic ischemic encephalopathy [12]. Similarly, tau has been identified to predict six-month outcomes regarding cerebral symptoms after cardiac arrest [13]. S100B and NSE have both been used as predictors for poor neurological outcome after traumatic brain injury [14]. S100B might enter the circulation after an isolated BBB injury with loss of BBB integrity, even without injury to the brain parenchyma [15].

Previous studies exploring cerebral biomarkers for preeclampsia have reported increased plasma concentrations before the onset of disease [16-18], during disease [19,20] and one year postpartum [21] in women with preeclampsia compared with women with normal pregnancies. Studies have also reported higher S100B plasma concentrations in women with severe preeclampsia and eclampsia compared with women with preeclampsia without severe features $[20,22]$. In addition, neurological symptoms, such as visual disturbances, have been correlated with increased S100B plasma concentrations [19,23].

The BBB is a highly restrictive and specialized neurovascular network that isolates and protects the brain parenchyma from potential harmful molecules present in the systemic circulation. Disruption of the BBB in preeclampsia and eclampsia has been studied in both laboratory and clinical settings, mainly with animal models [24], or ex vivo animal models exposed to plasma from women with preeclampsia. Cerebral edema, commonly seen in eclampsia and sometimes in severe preeclampsia, may partly be due to disruption of the BBB, resulting in increased BBB permeability and passage of fluid into the brain parenchyma $[25,26]$. Up until recently there has been a paucity of studies assessing the human BBB in preeclampsia, due to difficulties in studying the BBB in a clinical setting. However, our research group has recently presented promising results on the human cerebral microvascular endothelial cell line (hCMEC/D3) [27-29] as a new in vitro model of the BBB in preeclampsia research [30,31].

It is still not known if cerebral biomarkers are useful in reflecting BBB alterations in preeclampsia and to our knowledge, cerebral biomarkers have never before been correlated to any measures of BBB integrity in preeclampsia. Therefore, the aim of this study was to correlate plasma concentrations of cerebral biomarkers in women with preeclampsia, women with normal pregnancies and non-pregnant women with BBB integrity, measured as changes in transendothelial electrical resistance (TEER) in the hCMEC/D3 in vitro model. 


\section{Materials and Methods}

\subsection{Study Population}

The study population consisted of pregnant women with preeclampsia $(n=28)$ diagnosed according to the International Society for Studies on Hypertension in Pregnancy (ISSHP) 2018 guidelines [3]. Diagnostic criteria were de novo hypertension (systolic blood pressure $(\mathrm{SBP})>140$ and/or diastolic blood pressure $(\mathrm{DBP})>90 \mathrm{mmHg}$ ) in combination with significant proteinuria (protein level $>300 \mathrm{mg} / 24 \mathrm{~h}$ or urine dipstick $>1+$ ) after 20 gestational weeks. Although clinical preeclampsia can be diagnosed in the absence of proteinuria, with other signs of maternal organ dysfunction present, it has been recommended that proteinuria is used for patients enrolled in scientific research to ensure more specificity around the diagnosis [2]. These were also the common criteria used for preeclampsia diagnosis in the obstetric clinic at the time when cases were enrolled in the study.

Severe preeclampsia was defined according to the guidelines from the International Society for the study of Hypertension in Pregnancy (ISSHP) [32]. Criteria were an SBP $\geq 160$ and/or DBP $\geq 110$, development of HELLP syndrome, eclampsia or other severe organ manifestations.

As controls, women with normal pregnancies $(n=28)$, matched for maternal age and gestational length at inclusion, and non-pregnant women $(=16)$ were recruited. The definition of a normal pregnancy required that the woman remained normotensive throughout her pregnancy. The pregnancy also had to result in term delivery (gestational week $\geq 37$ ) of an infant with normal birth weight ( \pm 2 standard deviations of the mean birth weight for gestational age and sex) [33]. Women with prior hypertensive disorder in pregnancy were not included in any of the control groups. Further, none of the study groups included women with chronic hypertension, diabetes mellitus or chronic kidney disease.

The women were all recruited from the obstetric ward or the outpatient clinic at Uppsala University Hospital, Sweden between 2013 and 2016 [34].

Uppsala Ethical Review Board approved the study and informed consent was obtained from all participants.

\subsection{Sample Collection}

Plasma samples were collected in Vacutainer tubes with lithium heparin (Becton, Dickinson, Franklin Lakes, NJ, USA) within four hours of study inclusion. The samples were centrifuged for $10 \mathrm{~min}$ at $1500 \mathrm{~g}$ and the plasma was immediately frozen at $-70{ }^{\circ} \mathrm{C}$ for later analysis. Thawed plasma was used for analysis of the cerebral biomarkers. NfL and tau concentrations were measured in Mölndal, Sweden, whereas NSE and S100B concentrations were measured in Uppsala, Sweden. Further, frozen plasma was shipped to Chillán, Chile, thawed and added to the BBB model for TEER measurements.

\subsection{Biomarker Assay}

Plasma NfL concentration was measured with an in-house single molecule array (Simoa) method, whilst plasma tau concentration was measured with the Human Total Tau 2.0 kit and the Simoa platform (Quanterix, Billerica, MA, USA), both previously described in detail $[8,35]$. Laboratory technicians, who were blinded to clinical data, performed measurements in one round of experiments, using one batch of reagents. Two qualitycontrol samples were run in duplicates in the beginning and the end of each run, showing coefficients of variance (CVs) for intermediate precision of $6.0 \%$ at $8.5 \mathrm{pg} / \mathrm{mL}$ and $5.1 \%$ at $121 \mathrm{pg} / \mathrm{mL}$ for NfL, whereas CVs were $7.3 \%$ at $32.2 \mathrm{pg} / \mathrm{mL}$ and $7.0 \%$ at $7.5 \mathrm{pg} / \mathrm{mL}$ for tau.

Plasma NSE and S100B concentrations were measured by an enzyme-linked immunosorbent assay (ELISA). A commercially available kit (Sangtec 100 Elisa, Diasorin, MN, USA) was used and the samples were run according to the manufacturer's recommendation. The intra- and inter-assay coefficients of variation were $2.8 \%$ and $4.3 \%$ and $4.6 \%$ and $3.1 \%$, respectively, for NSE and S100B. 


\section{4. hCMEC/D3 In Vitro Model}

The hCMEC/D3 cell line (Merck Millipore, Darmstadt, Germany) [27] was used for the in vitro experiments. Monolayers of cells were seeded on semipermeable plates coated with rat-tail type I collagen (Discovery Labware, Bedford, MA, USA) at a density of 20,000 cells/well. Medium EndoGro MV Supplement Kit (Merck Millipore) was used as a culturing medium, and cells were incubated at $37^{\circ} \mathrm{C}, 5 \% \mathrm{CO}_{2}$. Once cells reached $100 \%$ of confluence, and a TEER value larger than $20 \Omega \mathrm{cm}^{2}$, they were used for experiments. In addition, six hours prior to experiments the culture medium was replaced by a medium without growth supplements. Cultured hCMEC/D3 cells were treated $(1 \%, v / v, 12 \mathrm{~h})$ with thawed plasma either from women with preeclampsia, women with normal pregnancies or non-pregnant women. An epithelial Volt/Ohm meter (EVOM2, World Precision Instruments, Sarasota, FL, USA) with two electrodes in each compartment was used to measure the TEER. Measurements were performed both before adding plasma (baseline), and after incubation with plasma. Delta-TEER values were calculated by subtracting basal TEER values from TEER values after exposure to plasma.

Confirmatory experiments of TEER and cell permeability to high-molecular weight fluorescent dye (Fluorescein-5-isothiocyanate FITC-dextran $70 \mathrm{kDa}$ ) were performed as previously reported [30] using randomly selected plasmas from women with preeclampsia $(n=12)$, and women with normal pregnancies $(n=13)$.

The hCMEC/D3 cell line was used in passages 5 to 10. Individual plasmas were used in duplicate experimental replicates. None of the plasmas were excluded at the final analysis. More detailed experimental conditions are described in a previous publication [30].

\subsection{Statistical Analyses}

Background characteristics were presented as medians with interquartile range (IQR) and numbers with percentage (\%) as appropriate. Groups were compared by one-way ANOVA, Chi-Square or Kruskal-Wallis test as appropriate, and the significance level was set at 0.05 .

Plasma concentrations of the cerebral biomarkers (NfL, tau, NSE and S100B), TEER and permeability were presented as medians with interquartile range (IQR). Differences between groups were compared by non-parametric analysis by Kruskal-Wallis test and pairwise comparisons by Mann-Whitney U-test. In case of statistical significance, a Bonferroni post-hoc test was used.

Associations between concentrations of the cerebral biomarkers NfL, tau, NSE and S100B, and TEER values were analyzed with a cumulative probability model [36] allowing for different, possibly non-linear, associations in the three groups of women. The model was further adjusted for baseline TEER, and for the confounders maternal age, parity and BMI. The confounders were identified with a DAG (directed acyclic graph). For this fully flexible model a $p$-value for all non-linear terms of 0.835 was calculated. The model was subsequently adjusted to a model where all associations were linear. Data and statistical analyses were performed with IBM SPSS Statistics for Windows, Version 25.0 (IBM Corp, Armonk, NY, USA), GraphPad Prism 6.00 (GraphPad Software, San Diego, CA, USA) and $R$ version 3.6.1 with the add-on package rms $[37,38]$.

Subgroup analyses of the women with preeclampsia were performed with regards to clinical symptoms in association with cerebral biomarkers and change in TEER, i.e., the difference between measured TEER before and after adding plasma to the model. Analyses were performed with the Mann-Whitney U-test.

\section{Results}

\subsection{Background Characteristics}

Clinical characteristics of the study population are described in Tables 1 and 2. There was no difference in maternal age or gestational length at inclusion between the pregnant study groups. Women with preeclampsia had a higher early-pregnancy body mass index (BMI) and were more often nulliparous compared with women with normal pregnancies 
and non-pregnant women. In the group of women with preeclampsia most women had antihypertensive treatment (79\%), and at inclusion approximately one third of them had severe headaches and/or visual disturbances. At delivery, $57 \%$ of the women had developed severe preeclampsia, with a recorded SBP $\geq 160$ and/or DBP $\geq 110$ [32]. None of the women developed eclampsia, neurological complications or other severe organ manifestations, and none required magnesium sulphate prophylaxis against imminent eclampsia.

Table 1. Clinical characteristics of the study population.

\begin{tabular}{|c|c|c|c|c|}
\hline Clinical Characteristics & Preeclampsia $(n=28)$ & Normal Pregnancy $(n=28)$ & Non-Pregnant $(n=16)$ & $p$-Values \\
\hline Maternal age (years) & $28(25-32)$ & $33(29-35)$ & $27(24-36)$ & n.s. \\
\hline Nulliparous & $23(82 \%)$ & $10(36 \%)$ & $9(56 \%)$ & $<0.001$ \\
\hline BMI & $26(23-29)$ & $24(22-26)$ & $22(20-25)$ & $<0.001$ \\
\hline \multicolumn{5}{|l|}{ At inclusion } \\
\hline Gestational week & $35(29-37)$ & $35(27-38)$ & & n.s. \\
\hline \multicolumn{5}{|c|}{ Blood pressure $(\mathrm{mmHg})$} \\
\hline Systolic & $150(140-160)$ & $110(110-120)$ & $110(110-118)$ & $<0.001$ \\
\hline Diastolic & $98(86-100)$ & $70(60-75)$ & $70(65-70)$ & $<0.001$ \\
\hline MAP & $113(107-120)$ & $83(77-90)$ & $83(80-86)$ & $<0.001$ \\
\hline \multicolumn{5}{|c|}{ Neurological symptoms (yes) } \\
\hline Headache & $18(64 \%)$ & $4(14 \%)$ & $3(19 \%)$ & $<0.001$ \\
\hline Severe headache (VAS $\geq 5$ ) & $10(36 \%)$ & 0 & 0 & $<0.001$ \\
\hline Visual disturbances & $10(36 \%)$ & 0 & 0 & $<0.001$ \\
\hline $\begin{array}{l}\text { Headache \& visual } \\
\text { disturbances }\end{array}$ & $10(36 \%)$ & 0 & 0 & $<0.001$ \\
\hline $\begin{array}{c}\text { Any neurological symptom } \\
\text { TEER }\left(\Omega \mathrm{cm}^{2}\right)\end{array}$ & $20(71 \%)$ & $4(14 \%)$ & $3(19 \%)$ & $<0.001$ \\
\hline Baseline value & $34.9(29.7-38.8)$ & $33.7(29.7-42.6)$ & $29.2(25.4-38.8)$ & n.s. \\
\hline After plasma exposure & $22.9(18.1-27.5)$ & $27.1(18.8-35.5)$ & $23.8(21.6-29.3)$ & n.s. \\
\hline$\Delta$-TEER & $11.9(8.5-14.8)$ & $7.6(3.7-11.9)$ & $5.8(2.0-8.0)$ & $<0.001$ \\
\hline
\end{tabular}

Data are presented as medians (IQR) or numbers (\%). Abbreviations: BMI, body mass index; MAP, mean arterial pressure; VAS, visual analogue scale; TEER, transendothelial electrical resistance; $\Delta$-TEER, the difference between TEER values before and after exposure to plasma; n.s., non-significant.

Table 2. Characteristics of the women with preeclampsia.

\begin{tabular}{cc}
\hline Clinical Characteristics & Preeclampsia $(\mathbf{n}=\mathbf{2 8})$ \\
\hline Gestational week at preeclampsia diagnosis & $35(22-41)$ \\
Severe preeclampsia at inclusion, $\mathrm{n}$ & $10(36 \%)$ \\
Blood pressure medication at inclusion, $\mathrm{n}$ & $22(79 \%)$ \\
Magnesium treatment, $\mathrm{n}$ & $0(0 \%)$ \\
Gestational week at delivery & $35(25-41)$ \\
Severe preeclampsia at delivery, $\mathrm{n}$ & $16(57 \%)$ \\
\hline
\end{tabular}

Numbers are presented as median (range) or numbers (\%). Severe preeclampsia is defined according to the guidelines from the International Society for the study of Hypertension in Pregnancy (ISSHP).

\subsection{Plasma Concentrations of NfL, Tau, NSE and S100B}

Compared with women with normal pregnancies, plasma concentrations of the cerebral biomarkers were higher in plasma from women with preeclampsia; NfL (5.25 ng/L, IQR 3.93-7.63 ng/L vs. $8.85 \mathrm{ng} / \mathrm{L}, \mathrm{IQR} 6.78-12.65 \mathrm{ng} / \mathrm{L}, p<0.001)$; tau $(2.40 \mathrm{ng} / \mathrm{L}$, IQR 1.80-2.58 ng/L vs. $2.90 \mathrm{ng} / \mathrm{L}, \mathrm{IQR} 2.40-4.35 \mathrm{ng} / \mathrm{L}, p<0.05)$; NSE $(2.37 \mu \mathrm{g} / \mathrm{L}, \mathrm{IQR}$ 1.93-2.85 $\mu \mathrm{g} / \mathrm{L}$ vs. $3.50 \mu \mathrm{g} / \mathrm{L}, \mathrm{IQR} 2.84-4.55 \mu \mathrm{g} / \mathrm{L}, p<0.001)$ and S100B (0.05 $\mu \mathrm{g} / \mathrm{L}, \mathrm{IQR}$ $0.03-0.08 \mu \mathrm{g} / \mathrm{L}$ vs. $0.08 \mu \mathrm{g} / \mathrm{L}, \mathrm{IQR} 0.05-0.10 \mu \mathrm{g} / \mathrm{L}, p<0.01)$ (Figure $1 \mathrm{a}-\mathrm{d}$ ). In addition, plasma concentrations of NfL were also higher in women with preeclampsia compared with non-pregnant women (8.85 ng/L, IQR 6.78-12.65 ng/L vs. $5.65 \mathrm{ng} / \mathrm{L}, \mathrm{IQR} 4.83-6.40 \mathrm{ng} / \mathrm{L}$, $p<0.001$ ) (Figure 1a). 
(a) NfL

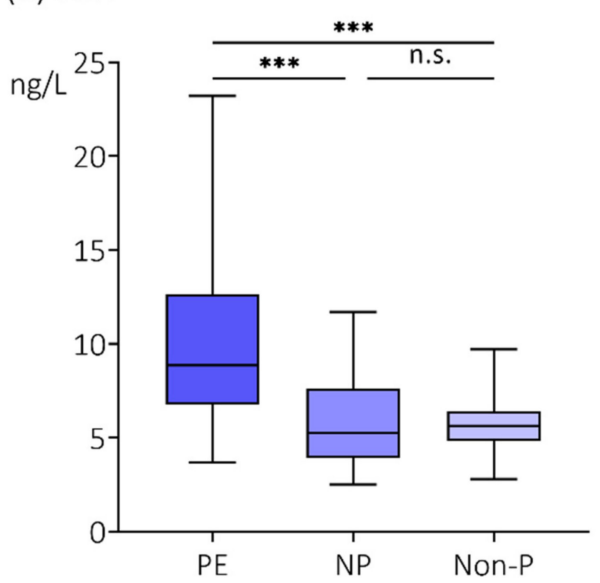

(c) NSE

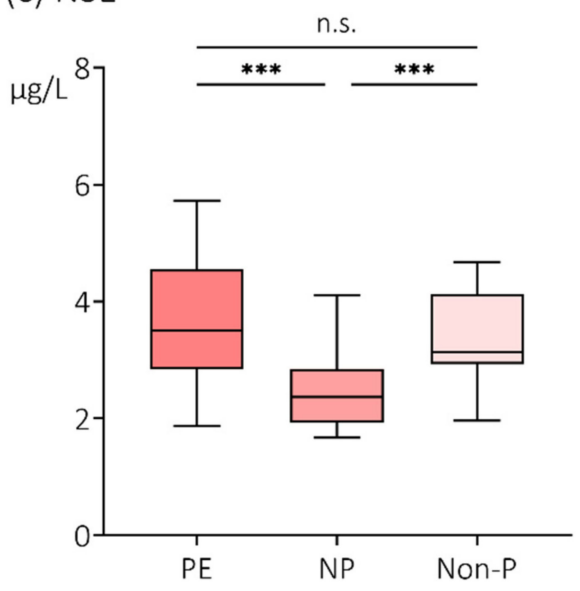

(b) tau

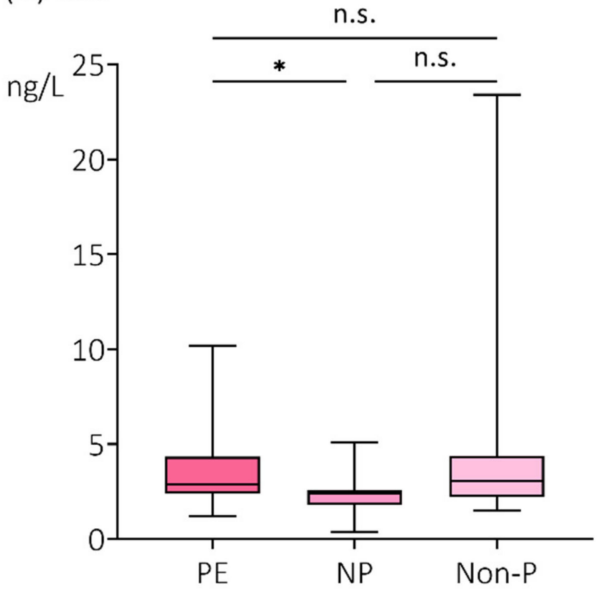

(d) S100B

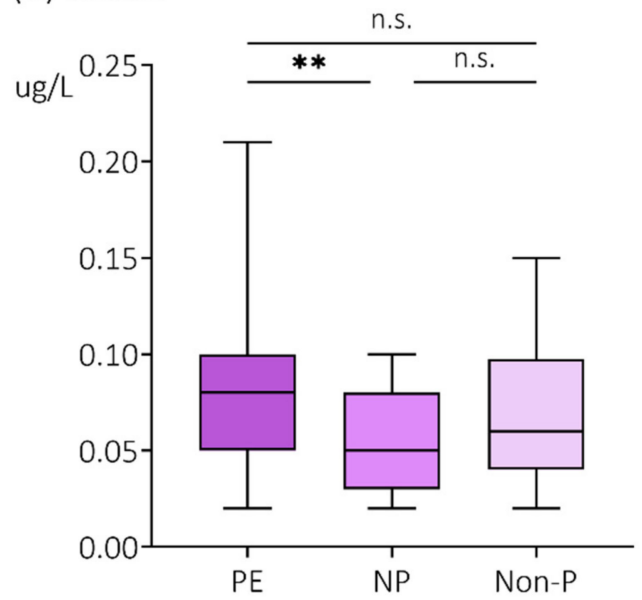

Figure 1. Plasma concentrations of (a) NfL, (b) tau, (c) NSE and (d) S100B. Plasma concentrations of the cerebral biomarkers (a) neurofilament light (NfL), (b) tau, (c) neuron specific enolase (NSE) and (d) S100B in women with preeclampsia (PE), women with normal pregnancies (NP) and nonpregnant women (Non-P). Values are represented by medians with interquartile range (IQR). Pairwise comparisons by Mann-Whitney U-test, Bonferroni correction. ${ }^{*} p<0.05$; ${ }^{* *} p<0.01$; ${ }^{* * *} p<0.001$; n.s. $=$ non-significant.

\subsection{Effects of Plasma on TEER in an In Vitro BBB Model}

The effects of plasma on changes in TEER, measured in the hCMEC/D3 in vitro model, in this population have been published previously [30]. Baseline TEER (i.e., TEER prior to exposure to plasma) was not statistically different among cell monolayers. After exposure to plasma, a significantly larger reduction in TEER was detected in cell monolayers exposed to plasma from women with preeclampsia compared with plasma from women with normal pregnancies and from non-pregnant women [30].

For this manuscript a re-confirmation analysis of previously published data was performed, where plasma was randomly included from the group of women with preeclampsia $(\mathrm{PE}, \mathrm{n}=12)$, and the women with normal pregnancies $(\mathrm{NP}, \mathrm{n}=13)$. Results are shown in Figure 2. 
(a) TEER

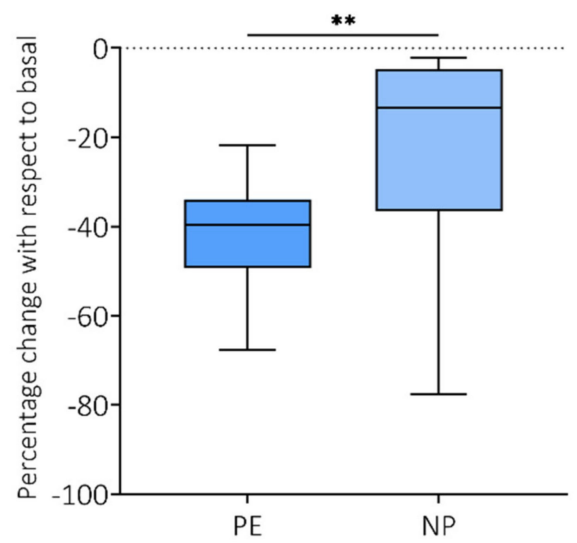

(b) Permeability

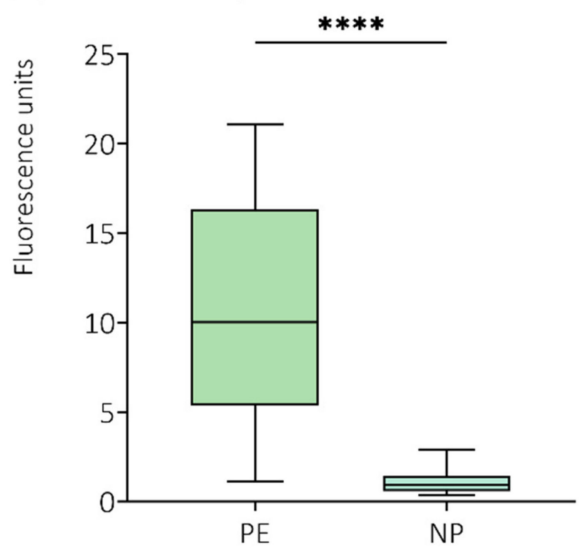

Figure 2. Re-confirmation analysis of TEER and permeability to $70 \mathrm{kDa}$ Dextran. Plasma from a small, randomly chosen sample of the women with preeclampsia (PE, $n=12)$ and the women with normal pregnancies (NP, $\mathrm{n}=13$ ) was analyzed for re-confirmation of previously published data on (a) transendothelial electrical resistance (TEER) and (b) permeability to $70 \mathrm{kDa}$ fluorescein isothiocyanate (FITC) - dextran [30]. ${ }^{* *} p<0.01$; ${ }^{* * *} p<0.0001$.

\subsection{Association of NfL, Tau, NSE and S100B with TEER}

The associations between cerebral biomarkers and TEER are presented in Figure 3. NfL was the only biomarker that showed a negative linear association with TEER, i.e., higher plasma concentrations of NfL correlated with a larger reduction in TEER $(p<0.01)$ after exposure to plasma. Data did not support associations between tau, NSE or S100B and TEER.

\subsection{Subgroup Analyses of Neurological Symptoms in the Women with Preeclampsia}

In the group of women with preeclampsia, 10 out of 28 women reported severe headaches (VAS $\geq 5$ ), and 10 women reported visual disturbances.

Higher plasma concentrations of NfL were found in the women with preeclampsia that expressed severe headache than in the women with mild or no headache (11.65 ng/L, IQR 9.58-15.63 ng/L vs. $7.40 \mathrm{ng} / \mathrm{L}$, IQR 5.93-9.93 ng/L, $p=0.024)$. The plasma concentrations of the other biomarkers (tau, NSE and S100B) did not relate to severity of headache. No associations were found between reported visual disturbances and any of the biomarkers. When comparing the women who had a combination of severe headache and visual disturbances with women who did not have this combination of symptoms, there were no differences in plasma concentrations of the respective biomarkers.

Women with preeclampsia that expressed severe headache (VAS $\geq 5)$ exhibited a greater reduction of TEER than the women with mild or no headache (10.17, IQR 7.32-10.45 vs. 7.74, IQR 5.65-9.42, $p=0.040$ ). No associations were found between reported visual disturbances and change in TEER. However, a greater reduction of TEER was seen in the women who had a combination of severe headache and visual disturbances, compared with the women without this combination of symptoms (10.32, IQR 7.82-11.72 vs. 7.81, IQR 5.77-9.56, $p<0.021$ ). 

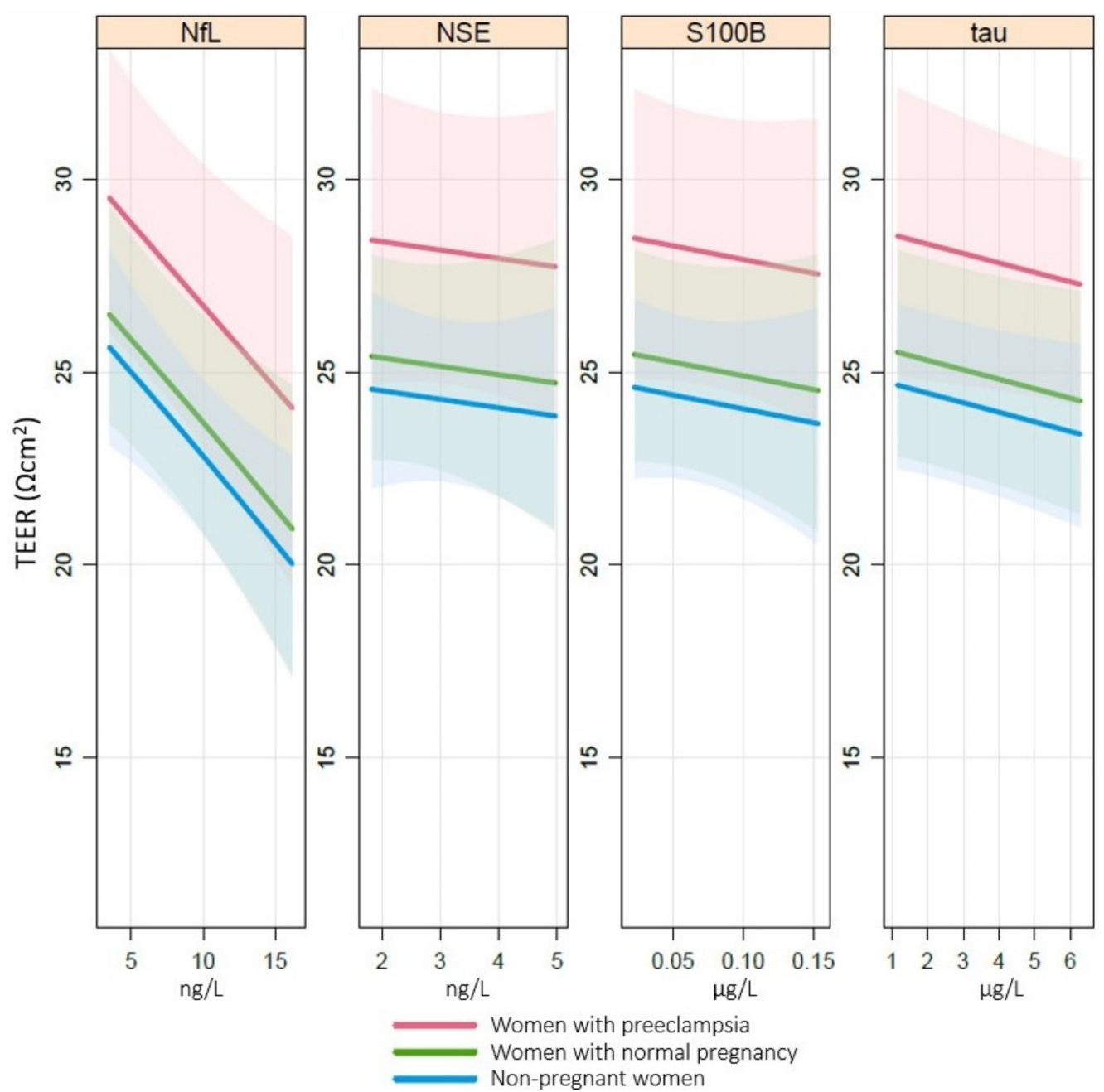

Figure 3. Association of TEER with NfL, tau, NSE and S100B. Associations between transendothelial electrical resistance (TEER $\left(\Omega \mathrm{cm}^{2}\right)$ ) and cerebral biomarkers neurofilament light (NfL (ng/L)), tau $(\mathrm{ng} / \mathrm{L})$, neuron-specific enolase (NSE $(\mu \mathrm{g} / \mathrm{L})$ ) and S100B $(\mu \mathrm{g} / \mathrm{L})$ in plasma analyzed by a cumulative probability model stratified by group and adjusted for baseline TEER, body mass index, parity and maternal age. NfL $p<0.01$, tau $p=$ n.s., NSE $p=$ n.s., S100B $p=$ n.s.

\section{Discussion}

\subsection{Main Findings}

In this study we demonstrated that circulating concentrations of NfL was associated with decreased TEER. In addition, we corroborated previous findings of higher plasma concentrations of the cerebral biomarkers NfL, tau, NSE and S100B in women with preeclampsia compared with women with normal pregnancies. Concentrations of NfL were also higher in women with preeclampsia compared with non-pregnant women. Further, women with preeclampsia that expressed severe headache had higher concentrations of plasma $\mathrm{NfL}$ and a greater reduction of TEER, compared with women with lesser symptoms. There was also a greater reduction of TEER in women with the combination of severe headache and visual disturbances.

\subsection{Research Implications}

Several previous studies have reported increased circulating concentrations of cerebral biomarkers in preeclampsia [16-18,20,22], findings that are also supported in this study. A novel finding, however, is that plasma concentrations of NfL were also significantly higher in plasma in women with preeclampsia compared with non-pregnant women. We did not 
find a difference in plasma concentrations of any of the biomarkers comparing women with normal pregnancies with non-pregnant women. Hence, pregnancy alone does not seem to have a major impact on circulating levels of these cerebral biomarkers.

NfL, tau, NSE and S100B have repeatedly been reported useful as circulating biomarkers of cerebral injury in other neurological disorders such as neurodegenerative disease, traumatic brain injury and epilepsy [8-10,14,15,39]. Previous studies of these biomarkers in relation to BBB injury have, however, most often evaluated biomarker concentrations in male or mixed populations with other neurological conditions than preeclampsia. The results from these studies seem to be somewhat conflicting regarding whether they support a BBB injury or not $[40,41]$. Our findings in a female population are suggestive of BBB alterations in terms of loss of ionic tightness, and possibly neuroaxonal injury, reflected by higher plasma concentrations of NfL in women with preeclampsia. We propose this by an association between higher circulating NfL concentrations in plasma and decreased TEER in an in vitro model of the BBB. However, this finding could have different underlying causes [15]. It could mean that intracerebral concentrations of NfL are normal, but are secreted over the BBB to a greater extent due to increased permeability, and are subsequently detected in higher concentrations in plasma. It could also reflect that NfL is released in higher concentrations in the central nervous system (CNS) following neuroaxonal injury, which might in turn cause the BBB to become more permeable or render increased NfL concentrations in plasma through alternative transport mehcanisms [15]. Alternatively, it may reflect a secondary breakdown of the BBB caused by circulating harmful molecules associated with preeclampsia, e.g., anti-angiogenic or inflammatory factors, including small extracellular vesicles [31], that allow NfL to cross from the brain to the circulation. It could also be caused by a combination of these proposed theories.

However, our findings could not support that higher plasma concentrations of NSE, S100B and tau had a significant association to decreased TEER. Thus, alternative explanations, such as other transport pathways across the BBB, dose-response-dependent passage, increased extracerebral existence or hemolysis in serum/plasma samples, have to be considered as causes of the higher concentrations of these biomarkers detected in women with preeclampsia. Furthermore, an in vitro model also has its own limitations and may not perfectly reflect in vivo conditions.

Recently published results from our research group reported increased concentrations of NfL in both plasma and CSF in women with preeclampsia compared with normotensive pregnant women. In addition, there was a strong correlation between concentrations of NfL in plasma and CSF [42]. Previous studies of women with preeclampsia have not been able to establish whether a BBB impairment exists in these women or not [43]; however, human studies are few on this subject.

Models for studying the brain and BBB function in preeclampsia that have found evidence of BBB injury have traditionally included in vitro models using animal endothelial cells, along with studies of brain hemodynamic regulation in humans and different preeclampsia-mimicking models in animals in vivo [24-26,29,44-46]. Recently our research group introduced the use of the hCMEC/D3 cell line as a means of studying the effect of preeclampsia on the BBB. We demonstrated a reduction of TEER, and increased permeability to $70 \mathrm{kDa}$ FITC-dextran over the BBB, when a monolayer of cells was exposed to plasma from women with preeclampsia and compared with plasma from women with normal pregnancies and non-pregnant controls [30]. The expression of tight junction proteins (zonula occludens-1 (ZO-1) and occludin) and phosphorylation of two tyrosine residues of VEGFR2 (pY951 and pY1175) were also explored, with no changes in the mRNA expression of the tight junction proteins. However, changes in their localization morphology or function were not investigated [30].

For this study we re-confirmed findings of a reduction in TEER and increased permeability in cells exposed to plasma from women with preeclampsia (Figure 2). In addition, we examined expression of tight junction protein claudin-5. This was performed as a sole experiment with only four randomly chosen plasma samples. Brain endothelial cells 
exposed to plasma from women with preeclampsia demonstrated a reduced protein abundance of claudin-5 in the cell membrane, whereas it was enhanced in the cytoplasmic fraction (Supplementary Material Figure S1).Characteristically, brain endothelial cells express higher levels of tight junction proteins than peripheral endothelial cells [47]. The key components of intercellular tight junctions are the transmembrane proteins occludin, claudin and junctional adhesion molecules (JAMs), which form complex strands that govern the permeability characteristics of the paracellular route [48,49]. Among them, claudins are directly responsible for the selective permeability of tight junctions [50,51]. There are 27 different claudin isoforms found in mammals [52]. Present in the cell membrane, claudin- 5 increases TEER, primarily by decreasing the permeability of cations [52,53]. In order to maintain this restricted diffusion pathway, tight junctions are linked to the cytoplasmic zonula occludens proteins that provide a structural bridge to the actin cytoskeleton. Consequently, the degree of tightness is determined by the interactions of tight junction family members on endothelial cells.

Pre-clinical studies show conflicting results regarding the involvement of these tight junction proteins on the increased BBB permeability seen in preeclampsia models [54-56]. One study found that despite exhibiting increased water content and increased BBB permeability in the anterior cerebrum in a preeclampsia-like syndrome in rats, the protein expression of claudin-1, ZO-1 and occludin was not altered in that part of the brain. However, an upregulation of claudin- 1 was detected in the posterior cerebrum of these animals, where brain water content was not altered [56].

In this manuscript, we report a reduction in the availability of claudin- 5 in the cell membrane of brain endothelial cells exposed to plasma from women with preeclampsia. This may constitute an underlying mechanism for the reduction in TEER under these experimental conditions.

To our knowledge, no previous studies have explored the association between circulating concentrations of cerebral biomarkers and BBB alterations in women with preeclampsia. However, several studies have established a correlation between serum or plasma concentrations of S100B, which as of now is the most explored of the cerebral biomarkers in preeclampsia, and severity of disease and/or neurological symptoms $[19,20,22,23]$.

\subsection{Strengths and Limitations}

Strengths in our study include the use of a human in vitro model of the BBB, as opposed to strictly animal-based models or the use of human plasma in animal models [24], to correlate BBB alterations in preeclampsia with peripheral biomarkers. This might reflect the human BBB to a higher extent compared with previous animal studies. Another strength is the well-characterized cohort of women and simultaneous measurements of cerebral biomarkers and BBB-assessment in the same woman.

A limitation of our study is that only one third of the women with preeclampsia in our cohort reported neurological symptoms, in the form of headaches or visual disturbances, and none developed severe cerebral complications [34]. The women in this study also underwent brain MRI scans, as part of previously published studies [34,57,58]. None of the women exhibited cerebral edema on their scans, which might reflect the fact that none of them suffered from severe neurological complications to preeclampsia, such as overt cerbral edema. Nevertheless, there were findings of decreased concentrations of magnesium $(\mathrm{Mg})$ on magnetic spectroscopy and decreased perfusion in the caudate nucleus in the women with preeclampsia. Since the study population did not fully reflect the degree of CNS pathophysiology that can occur in preeclampsia, our results are only generalizable to preeclampsia without severe neurological engagement.

Unfortunately, there were no CSF samples available for this population. Measurements of NfL in CSF, and also the CSF/serum albumin ratio, in the same women, could possibly have helped address what the plasma NfL finding represents: neuroaxonal injury and/or leakage across the BBB. 
For TEER measurements in this study we used a static in vitro model based on a monoculture. Future studies should include adaptations of the in vitro model to enhance the tightness of the BBB in order to better reflect in vivo conditions [27]. For example, co-culturing of hCMEC/D3 monolayers with astrocytes has been shown to increase TEER from 30 to $60 \Omega \mathrm{cm}^{2}$ [59], whereas exposure of hCMEC/D3 monolayers to a pulsatile flow in a capillary cartridge system resulted in TEER values of $1000-1200 \Omega \mathrm{cm}^{2}$ [60].

In this last regard, physical and technical parameters, such as setup, culture medium viscosity, electrodes and other instruments used, have been demonstrated to influence the measurement of TEER in BBB models. This may result in great variation of TEER results. Drawbacks of methods for TEER measurements have been further discussed by Vigh et al. [61]. To avoid these potential experimental pitfalls, all in vitro experiments were run in a blinded manner for the groups of plasma. In addition, analyses were performed head-to-head using plasmas from the three different groups, as previously reported [30].

\subsection{Future Perspectives}

Manifestations of acute cerebral complications of preeclampsia, such as eclampsia and cerebral hemorrhage or edema, are difficult to predict. A circulating biomarker, alone or in combination with other clinical data, that could identify women with preeclampsia at risk of such events, may in the future contribute to the prevention of severe cerebral complications. This would allow for a more customized management of women at high risk, e.g., by suggesting more aggressive treatment with antihypertensive drugs, administration of neuroprotective treatment such as $\mathrm{MgSO}_{4}$ [46], and/or aid in decisions about timing of delivery. Here we suggest that NfL could be a promising biomarker for BBB alterations and/or axonal injury in preeclampsia. However, it needs to be further explored in a population presenting with cerebral complications of the disease, and additional methods to assess BBB permeability and function should also be considered.

\section{Conclusions}

We demonstrated that plasma concentrations of NfL had a negative association with TEER in an in vitro model of the BBB. This finding supports the role of NfL as a promising biomarker to reflect cerebral involvement in women with preeclampsia. Further, in women with preeclampsia we found that those with severe headache, a possible sign of cerebral involvement, had both higher concentrations of plasma NfL and a greater reduction of TEER, compared with those without severe headache.

Prospective studies should be conducted to confirm the role of NfL as a biomarker for BBB alterations and/or neuroaxonal injury in women with preeclampsia, preferably in a population with manifest neurological complications.

Supplementary Materials: The following supporting information can be downloaded at: https:// www.mdpi.com/article/10.3390/cells11050789/s1, Figure S1: Reduced protein abundance of claudin5 in cell membrane of brain endothelial cells exposed to plasma from women with preeclampsia [62].

Author Contributions: C.E. and L.B. conceptualized the study. L.B., T.F., C.E. and A.-K.W. conducted the statistical analyses. T.F., L.B., A.-K.W. and C.E. wrote the manuscript. M.N. collected the data. J.A. and J.L. performed the BBB experiments. P.T.-V. was a consultant for the in vitro BBB experiments. H.Z., K.B., H.Å. and H.K. performed the biomarker analyses. F.T. performed confirmatory experiments regarding TEER and permeability, and claudin-5. C.C. helped supervise the project. All authors have read and agreed to the published version of the manuscript.

Funding: This research was funded by the Swedish foundation for international cooperation in research and higher education (STINT) for collaboration between Chile and Sweden (Saving maternal brains; MG2019-8462); The Swedish Research Council (Saving maternal brains; 2020-01640); The Swedish Brain Foundation (Saving maternal brains; FO2019-0128). L.B. is supported by the Swedish Society of Medicine (SLS-934559). C.E. is financially supported by Fondecyt (1200250). T.F. received funding from the Birth Foundation. 
Institutional Review Board Statement: Ethical approval was obtained from Uppsala Ethical Review Board (protocol number 2012/087, approval of amendment: protocol number 2012/087/1).

Informed Consent Statement: Informed consent was obtained from all subjects involved in the study.

Data Availability Statement: The data presented in this study will be anonymized and made available on request from the corresponding author, after approval from the Uppsala Ethical Review Board. The data are not publicly available due to secrecy.

Acknowledgments: The authors would like to thank the researchers from GRIVAS health for their valuable input. We would also like to thank Erik Lampa, Uppsala Clinical Research Center, for valuable statistical support.

Conflicts of Interest: H.Z. has served at scientific advisory boards and/or as a consultant for Abbvie, Alector, Eisai, Denali, Roche, Wave, Samumed, Siemens Healthineers, Pinteon Therapeutics, Nervgen, AZTherapies, CogRx and Red Abbey Labs, has given lectures in symposia sponsored by Cellectricon, Fujirebio, Alzecure and Biogen, and is a co-founder of Brain Biomarker Solutions in Gothenburg AB (BBS), which is a part of the GU Ventures Incubator Program. The funders had no role in the design of the study; in the collection, analyses, or interpretation of data; in the writing of the manuscript, or in the decision to publish the results. The other authors declare no conflict of interest.

\section{References}

1. Chappell, L.C.; Cluver, C.A.; Kingdom, J.; Tong, S. Pre-eclampsia. Lancet 2021, 398, 341-354. [CrossRef]

2. Tranquilli, A.L.; Dekker, G.; Magee, L.; Roberts, J.; Sibai, B.M.; Steyn, W.; Zeeman, G.G.; Brown, M.A. The classification, diagnosis and management of the hypertensive disorders of pregnancy: A revised statement from the ISSHP. Pregnancy Hypertens. 2014, 4, 97-104. [CrossRef] [PubMed]

3. Brown, M.A.; Magee, L.A.; Kenny, L.C.; Karumanchi, S.A.; McCarthy, F.P.; Saito, S.; Hall, D.R.; Warren, C.E.; Adoyi, G.; Ishaku, S.; et al. Hypertensive Disorders of Pregnancy: ISSHP Classification, Diagnosis, and Management Recommendations for International Practice. Hypertension 2018, 72, 24-43. [CrossRef] [PubMed]

4. MacKay, A.P.; Berg, C.J.; Atrash, H.K. Pregnancy-related mortality from preeclampsia and eclampsia. Obstet. Gynecol. 2001, 97, 533-538. [CrossRef] [PubMed]

5. Say, L.; Chou, D.; Gemmill, A.; Tuncalp, O.; Moller, A.B.; Daniels, J.; Gulmezoglu, A.M.; Temmerman, M.; Alkema, L. Global causes of maternal death: A WHO systematic analysis. Lancet Glob. Health 2014, 2, e323-e333. [CrossRef]

6. Hastie, R.; Brownfoot, F.C.; Cluver, C.A.; Walker, S.P.; Hesselman, S.; Tong, S.; Bergman, L. Predictive Value of the Signs and Symptoms Preceding Eclampsia: A Systematic Review. Obstet. Gynecol. 2019. [CrossRef]

7. Donato, R.; Cannon, B.R.; Sorci, G.; Riuzzi, F.; Hsu, K.; Weber, D.J.; Geczy, C.L. Functions of S100 proteins. Curr. Mol. Med. 2013, 13, 24-57. [CrossRef]

8. Rohrer, J.D.; Woollacott, I.O.; Dick, K.M.; Brotherhood, E.; Gordon, E.; Fellows, A.; Toombs, J.; Druyeh, R.; Cardoso, M.J.; Ourselin, S.; et al. Serum neurofilament light chain protein is a measure of disease intensity in frontotemporal dementia. Neurology 2016, 87, 1329-1336. [CrossRef]

9. Avila, J.; Lucas, J.J.; Perez, M.; Hernandez, F. Role of tau protein in both physiological and pathological conditions. Physiol. Rev. 2004, 84, 361-384. [CrossRef]

10. Bogoslovsky, T.; Gill, J.; Jeromin, A.; Davis, C.; Diaz-Arrastia, R. Fluid Biomarkers of Traumatic Brain Injury and Intended Context of Use. Diagnostics 2016, 6, 37. [CrossRef]

11. Marchi, N.; Rasmussen, P.; Kapural, M.; Fazio, V.; Kight, K.; Mayberg, M.R.; Kanner, A.; Ayumar, B.; Albensi, B.; Cavaglia, M.; et al. Peripheral markers of brain damage and blood-brain barrier dysfunction. Restor. Neurol. Neurosci. 2003, 21, 109-121. [PubMed]

12. Chou, S.H.; Robertson, C.S.; the Participants in the International Multi-disciplinary Consensus Conference on the Multimodality Monitoring. Monitoring biomarkers of cellular injury and death in acute brain injury. Neurocrit. Care 2014, 21 (Suppl. S2), S187-S214. [CrossRef] [PubMed]

13. Randall, J.; Mortberg, E.; Provuncher, G.K.; Fournier, D.R.; Duffy, D.C.; Rubertsson, S.; Blennow, K.; Zetterberg, H.; Wilson, D.H. Tau proteins in serum predict neurological outcome after hypoxic brain injury from cardiac arrest: Results of a pilot study. Resuscitation 2013, 84, 351-356. [CrossRef] [PubMed]

14. Stefanovic, B.; Duric, O.; Stankovic, S.; Mijatovic, S.; Doklestic, K.; Stefanovic, B.; Jovanovic, B.; Marjanovic, N.; Kalezic, N. Elevated Serum Protein S100B and Neuron Specific Enolase Values as Predictors of Early Neurological Outcome After Traumatic Brain Injury. J. Med. Biochem. 2017, 36, 314-321. [CrossRef]

15. Marchi, N.; Cavaglia, M.; Fazio, V.; Bhudia, S.; Hallene, K.; Janigro, D. Peripheral markers of blood-brain barrier damage. Clin. Chim. Acta 2004, 342, 1-12. [CrossRef]

16. Evers, K.S.; Atkinson, A.; Barro, C.; Fisch, U.; Pfister, M.; Huhn, E.A.; Lapaire, O.; Kuhle, J.; Wellmann, S. Neurofilament as Neuronal Injury Blood Marker in Preeclampsia. Hypertension 2018, 71, 1178-1184. [CrossRef] 
17. Bergman, L.; Zetterberg, H.; Kaihola, H.; Hagberg, H.; Blennow, K.; Akerud, H. Blood-based cerebral biomarkers in preeclampsia: Plasma concentrations of NfL, tau, S100B and NSE during pregnancy in women who later develop preeclampsia-A nested case control study. PLoS ONE 2018, 13, e0196025. [CrossRef]

18. Bergman, L.; Akerud, H. Plasma Levels of the Cerebral Biomarker, Neuron-Specific Enolase, are Elevated During Pregnancy in Women Developing Preeclampsia. Reprod. Sci. 2016, 23, 395-400. [CrossRef]

19. Bergman, L.; Akhter, T.; Wikstrom, A.K.; Wikstrom, J.; Naessen, T.; Akerud, H. Plasma levels of S100B in preeclampsia and association with possible central nervous system effects. Am. J. Hypertens. 2014, 27, 1105-1111. [CrossRef]

20. Vettorazzi, J.; Torres, F.V.; de Avila, T.T.; Martins-Costa, S.H.; Souza, D.O.; Portela, L.V.; Ramos, J.G. Serum S100B in pregnancy complicated by preeclampsia: A case-control study. Pregnancy Hypertens. 2012, 2, 101-105. [CrossRef]

21. Bergman, L.; Akerud, H.; Wikstrom, A.K.; Larsson, M.; Naessen, T.; Akhter, T. Cerebral Biomarkers in Women With Preeclampsia Are Still Elevated 1 Year Postpartum. Am. J. Hypertens. 2016, 29, 1374-1379. [CrossRef] [PubMed]

22. Schmidt, A.P.; Tort, A.B.; Amaral, O.B.; Schmidt, A.P.; Walz, R.; Vettorazzi-Stuckzynski, J.; Martins-Costa, S.H.; Ramos, J.G.; Souza, D.O.; Portela, L.V. Serum S100B in pregnancy-related hypertensive disorders: A case-control study. Clin. Chem. 2004, 50, 435-438. [CrossRef] [PubMed]

23. Artunc-Ulkumen, B.; Guvenc, Y.; Goker, A.; Gozukara, C. Maternal Serum S100-B, PAPP-A and IL-6 levels in severe preeclampsia Arch. Gynecol Obs. 2015, 292, 97-102. [CrossRef] [PubMed]

24. Cipolla, M.J. Cerebrovascular function in pregnancy and eclampsia. Hypertension 2007, 50, 14-24. [CrossRef]

25. Hammer, E.S.; Cipolla, M.J. Cerebrovascular Dysfunction in Preeclamptic Pregnancies. Curr. Hypertens. Rep. 2015, 17, 64. [CrossRef]

26. Amburgey, O.A.; Chapman, A.C.; May, V.; Bernstein, I.M.; Cipolla, M.J. Plasma from preeclamptic women increases blood-brain barrier permeability: Role of vascular endothelial growth factor signaling. Hypertension 2010, 56, 1003-1008. [CrossRef]

27. Weksler, B.; Romero, I.A.; Couraud, P.O. The hCMEC/D3 cell line as a model of the human blood brain barrier. Fluids Barriers CNS 2013, 10, 16. [CrossRef]

28. Rahman, N.A.; Rasil, A.; Meyding-Lamade, U.; Craemer, E.M.; Diah, S.; Tuah, A.A.; Muharram, S.H. Immortalized endothelial cell lines for in vitro blood-brain barrier models: A systematic review. Brain Res. 2016, 1642, 532-545. [CrossRef]

29. Deli, M.A.; Abraham, C.S.; Kataoka, Y.; Niwa, M. Permeability studies on in vitro blood-brain barrier models: Physiology, pathology, and pharmacology. Cell. Mol. Neurobiol. 2005, 25, 59-127. [CrossRef]

30. Bergman, L.; Acurio, J.; Leon, J.; Gatu, E.; Friis, T.; Nelander, M.; Wikstrom, J.; Larsson, A.; Lara, E.; Aguayo, C.; et al. Preeclampsia and increased permeability over the blood brain barrier-A role of vascular endothelial growth receptor 2. Am. J. Hypertens. 2020 [CrossRef]

31. León, J.; Acurio, J.; Bergman, L.; López, J.; Wikström, A.-K.; Torres-Vergara, P.; Troncoso, F.; Castro, F.O.; Vatish, M.; Escudero, C. Disruption of the Blood-Brain Barrier by Extracellular Vesicles From Preeclampsia Plasma and Hypoxic Placentae: Attenuation by Magnesium Sulfate. Hypertension 2021. [CrossRef]

32. Tranquilli, A.L.; Brown, M.A.; Zeeman, G.G.; Dekker, G.; Sibai, B.M. The definition of severe and early-onset preeclampsia. Statements from the International Society for the Study of Hypertension in Pregnancy (ISSHP). Pregnancy Hypertens. 2013, 3 , 44-47. [CrossRef] [PubMed]

33. Marsal, K.; Persson, P.H.; Larsen, T.; Lilja, H.; Selbing, A.; Sultan, B. Intrauterine growth curves based on ultrasonically estimated foetal weights. Acta Paediatr. 1996, 85, 843-848. [CrossRef] [PubMed]

34. Nelander, M.; Weis, J.; Bergman, L.; Larsson, A.; Wikstrom, A.K.; Wikstrom, J. Cerebral Magnesium Levels in Preeclampsia; A Phosphorus Magnetic Resonance Spectroscopy Study. Am. J. Hypertens. 2017, 30, 667-672. [CrossRef]

35. Mattsson, N.; Zetterberg, H.; Janelidze, S.; Insel, P.S.; Andreasson, U.; Stomrud, E.; Palmqvist, S.; Baker, D.; Tan Hehir, C.A.; Jeromin, A.; et al. Plasma tau in Alzheimer disease. Neurology 2016, 87, 1827-1835. [CrossRef] [PubMed]

36. Liu, Q.; Shepherd, B.E.; Li, C.; Harrell, F.E., Jr. Modeling continuous response variables using ordinal regression. Stat. Med. 2017, 36, 4316-4335. [CrossRef] [PubMed]

37. R: A Language and Environment for Statistical Computing. Available online: https://www.R-project.org/ (accessed on 26 December 2021).

38. Harrell, F.E., Jr. rms: Regression Modeling Strategies. R Package Version 6.2-0. Available online: https: / /CRAN.R-project.org/ package $=$ rms (accessed on 26 December 2021).

39. Simani, L.; Sadeghi, M.; Ryan, F.; Dehghani, M.; Niknazar, S. Elevated Blood-Based Brain Biomarker Levels in Patients with Epileptic Seizures: A Systematic Review and Meta-analysis. ACS Chem. Neurosci. 2020, 11, 4048-4059. [CrossRef]

40. Kalm, M.; Bostrom, M.; Sandelius, A.; Eriksson, Y.; Ek, C.J.; Blennow, K.; Bjork-Eriksson, T.; Zetterberg, H. Serum concentrations of the axonal injury marker neurofilament light protein are not influenced by blood-brain barrier permeability. Brain Res. 2017, 1668, 12-19. [CrossRef]

41. Uher, T.; McComb, M.; Galkin, S.; Srpova, B.; Oechtering, J.; Barro, C.; Tyblova, M.; Bergsland, N.; Krasensky, J.; Dwyer, M.; et al. Neurofilament levels are associated with blood-brain barrier integrity, lymphocyte extravasation, and risk factors following the first demyelinating event in multiple sclerosis. Mult. Scler. 2021, 27, 220-231. [CrossRef]

42. Andersson, M.; Oras, J.; Thorn, S.E.; Karlsson, O.; Kalebo, P.; Zetterberg, H.; Blennow, K.; Bergman, L. Signs of neuroaxonal injury in preeclampsia-A case control study. PLoS ONE 2021, 16, e0246786. [CrossRef] 
43. Burwick, R.M.; Togioka, B.M.; Speranza, R.J.; Gaffney, J.E.; Roberts, V.H.J.; Frias, A.E.; Rincon, M. Assessment of blood-brain barrier integrity and neuroinflammation in preeclampsia. Am. J. Obs. Gynecol. 2019, 221, 269.e1-269.e8. [CrossRef] [PubMed]

44. Helms, H.C.; Abbott, N.J.; Burek, M.; Cecchelli, R.; Couraud, P.O.; Deli, M.A.; Forster, C.; Galla, H.J.; Romero, I.A.; Shusta, E.V.; et al. In vitro models of the blood-brain barrier: An overview of commonly used brain endothelial cell culture models and guidelines for their use. J. Cereb. Blood Flow Metab. 2016, 36, 862-890. [CrossRef] [PubMed]

45. van Veen, T.R.; Panerai, R.B.; Haeri, S.; Singh, J.; Adusumalli, J.A.; Zeeman, G.G.; Belfort, M.A. Cerebral autoregulation in different hypertensive disorders of pregnancy. Am. J. Obs. Gynecol. 2015, 212, 513.e1-513.e7. [CrossRef] [PubMed]

46. Johnson, A.C.; Tremble, S.M.; Chan, S.L.; Moseley, J.; LaMarca, B.; Nagle, K.J.; Cipolla, M.J. Magnesium sulfate treatment reverses seizure susceptibility and decreases neuroinflammation in a rat model of severe preeclampsia. PLoS ONE 2014, 9, e113670. [CrossRef]

47. Uwamori, H.; Ono, Y.; Yamashita, T.; Arai, K.; Sudo, R. Comparison of organ-specific endothelial cells in terms of microvascular formation and endothelial barrier functions. Microvasc. Res. 2019, 122, 60-70. [CrossRef] [PubMed]

48. Haseloff, R.F.; Dithmer, S.; Winkler, L.; Wolburg, H.; Blasig, I.E. Transmembrane proteins of the tight junctions at the blood-brain barrier: Structural and functional aspects. Semin. Cell Dev. Biol. 2015, 38, 16-25. [CrossRef]

49. Keaney, J.; Campbell, M. The dynamic blood-brain barrier. FEBS J. 2015, 282, 4067-4079. [CrossRef]

50. Koval, M. Claudins-Key pieces in the tight junction puzzle. Cell Commun. Adhes. 2006, 13, 127-138. [CrossRef]

51. Krause, G.; Winkler, L.; Mueller, S.L.; Haseloff, R.F.; Piontek, J.; Blasig, I.E. Structure and function of claudins. Biochim. Biophys. Acta 2008, 1778, 631-645. [CrossRef]

52. Angelow, S.; Ahlstrom, R.; Yu, A.S. Biology of claudins. Am. J. Physiol. Ren. Physiol. 2008, 295, F867-F876. [CrossRef]

53. Wen, H.; Watry, D.D.; Marcondes, M.C.; Fox, H.S. Selective decrease in paracellular conductance of tight junctions: Role of the first extracellular domain of claudin-5. Mol. Cell Biol. 2004, 24, 8408-8417. [CrossRef] [PubMed]

54. Marra, A.; Vargas, M.; Striano, P.; Del Guercio, L.; Buonanno, P.; Servillo, G. Posterior reversible encephalopathy syndrome: The endothelial hypotheses. Med. Hypotheses. 2014, 82, 619-622. [CrossRef] [PubMed]

55. Clayton, A.M.; Shao, Q.; Paauw, N.D.; Giambrone, A.B.; Granger, J.P.; Warrington, J.P. Postpartum increases in cerebral edema and inflammation in response to placental ischemia during pregnancy. Brain Behav. Immun. 2018, 70, 376-389. [CrossRef]

56. Warrington, J.P.; Fan, F.; Murphy, S.R.; Roman, R.J.; Drummond, H.A.; Granger, J.P.; Ryan, M.J. Placental ischemia in pregnant rats impairs cerebral blood flow autoregulation and increases blood-brain barrier permeability. Physiol. Rep. 2014, 2. [CrossRef] [PubMed]

57. Nelander, M.; Hannsberger, D.; Sundström-Poromaa, I.; Bergman, L.; Weis, J.; Åkerud, H.; Wikström, J.; Wikström, A.-K. Assessment of cerebral perfusion and edema in preeclampsia with intravoxel incoherent motion MRI. AOGS 2018. [CrossRef]

58. Nelander, M.; Wikström, A.-K.; Weis, J.; Bergman, L.; Larsson, A.; Sundström-Poromaa, I.; Wikström, J. Cerebral Osmolytes and Plasma Osmolality in Pregnancy and Preeclampsia: A Proton Magnetic Resonance Spectroscopy Study. Am. J. Hypertens. 2018, 31, 847-853. [CrossRef]

59. Hatherell, K.; Couraud, P.O.; Romero, I.A.; Weksler, B.; Pilkington, G.J. Development of a three-dimensional, all-human in vitro model of the blood-brain barrier using mono-, co-, and tri-cultivation Transwell models. J. Neurosci. Methods 2011, 199, 223-229. [CrossRef]

60. Cucullo, L.; Couraud, P.O.; Weksler, B.; Romero, I.A.; Hossain, M.; Rapp, E.; Janigro, D. Immortalized human brain endothelial cells and flow-based vascular modeling: A marriage of convenience for rational neurovascular studies. J. Cereb. Blood Flow Metab. 2008, 28, 312-328. [CrossRef]

61. Vigh, J.P.; Kincses, A.; Ozgür, B.; Walter, F.R.; Santa-Maria, A.R.; Valkai, S.; Vastag, M.; Neuhaus, W.; Brodin, B.; Dér, A. Transendothelial Electrical Resistance Measurement across the Blood-Brain Barrier: A Critical Review of Methods. Micromachines 2021, 12, 685. [CrossRef]

62. Escudero, C.; Bertoglia, P.; Hernadez, M.; Celis, C.; Gonzalez, M.; Aguayo, C.; Acurio, J. Impaired A2A adenosine receptor/nitric oxide/VEGF signaling pathway in fetal endothelium during late- and early-onset preeclampsia. Purinergic Signal. 2013, 9, 215-226. [CrossRef] 